\title{
Perspektive Vielfalt
}

VON WOLFGANG SCHMIDT

UND CLAUDIA DE FRIES

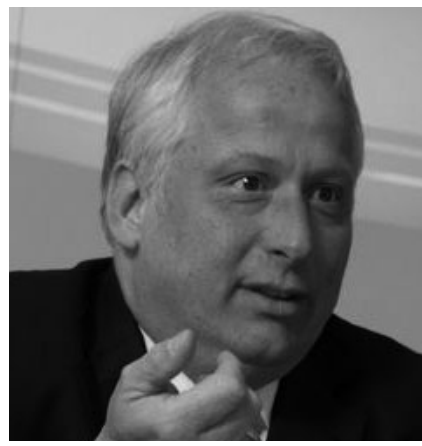

Wolfgang Schmidt ist Geschäftsführer der Kinder - und Familienhilfen Michaelshoven gemeinnützige $\mathrm{GmbH}$ und des Bereiches: Die Sozialen Hilfen e.V. Er ist Diversitymanager und verantwortlich für das Projekt "Perspektive Vielfalt» der Diakonie Michaelshoven. w.schmidt@diakonie-michaelshoven.de

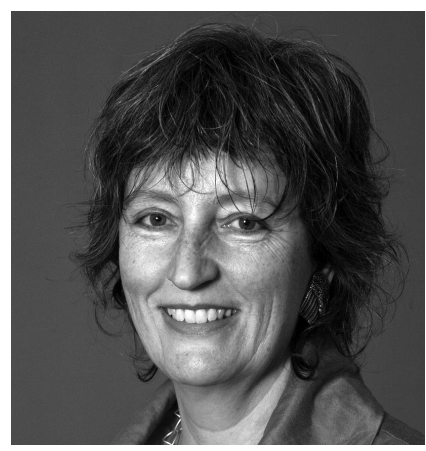

Claudia de Fries ist Prozessbegleiterin für das Projekt "Perspektive Vielfalt« und verantwortlich für den Bereich Personal: Gewinnung und Entwicklung im Geschäftsfeld der Kinder- und Familienhilfen gGmbH sowie Die Sozialen Hilfen e. $\mathrm{V}$.

c.defries@diakonie-michaelshoven.de

\section{Engagement für mehr Gesundheit senkt} Krankenstände sowie Fluktuation und steigert die Mitarbeiterzufriedenheit. Das Betriebliche Gesundheitsmanagement bietet ein System von Strukturen, Prozessen und Rahmenbedingungen, welches auch das Problem der knapper werdenden Ressourcen und demografischen Veränderungen berücksichtigt, wie das Beispiel der Diakonie Michaelhoven in Nordrhein-Westfalen zeigt.

Das Thema Gesundheitsförderung ist in Zeiten des aufkommenden Fachkräftemangels eines, das auf der Agenda Führungsverantwortlicher in sozialen Unternehmen inzwischen auf einen der vorderen Plätze gehoben wurde. Unter den Überschriften »Personalbindung « und »attraktiver Arbeitgeber" spielen Angebote für die Förderung und den Erhalt der Gesundheit der Mitarbeitenden eine entscheidende Rolle. Aber auch aus dem Blickwinkel des Diversity-Managements ist die Gesundheit von Mitarbeitenden eine Dimension, die nicht ignoriert werden sollte. So auch bei der Diakonie Michaelshoven (DM), die sich seit mehreren Jahren mit dem DiversityManagement und seinen Teilaspekten beschäftigt.

\section{Der Diversity-Ansatz der Diakonie Michaelshoven}

Ausgangspunkt für die Diversity-Überlegungen in der Diakonie Michaelshoven sind folgende Grundannahmen, die gleichzeitig Motor und Ziel bedeuten:

- Diversity Management wird im Sinne von »soziale Vielfalt konstruktiv nutzen« verwendet.

- Diversity Management toleriert nicht nur die individuelle Verschiedenheit (engl.: diversity) der Mitarbeitenden, sondern hebt diese im Sinne einer positiven Wertschätzung besonders hervor.

- Ziele von Diversity Management sind es, eine produktive Gesamtatmosphäre im Unternehmen zu erreichen, soziale Diskriminierungen von Minderheiten zu verhindern und Chancengleichheit zu verbessern.

Wenn diese Grundannahmen ernst genommen werden, ist es nicht mehr weit zu einer positiven Unternehmenskultur und damit einhergehend Maßnahmen und Angeboten, die ein wertschätzendes Klima nicht nur Anspruch, sondern Wirklichkeit werden lassen. Auf dem Weg der Implementierung hat das Diversity-Management in der Diakonie Michaelshoven bereits einige Meilensteine hinter sich lassen können (vgl. Kasten).

»Perspektive Vielfalt « erhält zur Unterstützung, Koordination und Begleitung von Maßnahmen seit 2011 bis voraussichtlich Ende 2013 eine personelle Ressource in Form einer Prozessbegleitung befristet zur Verfügung. Ein weiteres essenzielles Element ist die Beauftragung durch und Rückbindung an das oberste Leitungsgremium des Unternehmens (Geschäftsführer und Vorstand), um die Aktivitäten der »Vielfaltsbeauftragten « aus den Geschäftsfeldern und der zugehörigen Begleitgruppe Vielfalt immer wieder an aktuelle strategische Erfordernisse anzupassen. 


\begin{tabular}{|c|c|c|c|}
\hline Maßnahme & Seite & Instrument & Seite \\
\hline \multicolumn{4}{|l|}{ Gesundheit und Leistungsfähigkeit } \\
\hline Förderung einer Gesundheitskultur & 55 & Gesundheitstag & 57 \\
\hline $\begin{array}{l}\text { Ergonomische Arbeitsgestaltung } \\
\text { (Verhältnisprävention) }\end{array}$ & 59 & $\begin{array}{l}\text { Gestaltung der Arbeitsplätze älterer } \\
\text { Mitarbeiter/-innen }\end{array}$ & 61 \\
\hline $\begin{array}{l}\text { Förderung gesundheitlichen Verhaltens: } \\
\text { Verhältnisprävention }\end{array}$ & 63 & $\begin{array}{l}\text { Stressmanagement } \\
\text { Rückenschule }\end{array}$ & $\begin{array}{l}65 \\
67\end{array}$ \\
\hline $\begin{array}{l}\text { Stärkung der individuellen Gesundheits- } \\
\text { kompetenz }\end{array}$ & 69 & Gesundheitsschecks & 71 \\
\hline $\begin{array}{l}\text { Umsetzung gesetzlicher Vorschriften } \\
\text { zum Arbeits- und Gesundheitsschutz }\end{array}$ & 73 & $\begin{array}{l}\text { Schulung von Beschäftigten und } \\
\text { Führungskräften }\end{array}$ & 75 \\
\hline Betriebliches Gesundheitsmanagement & 77 & Gesundheitszirkel & 79 \\
\hline \multicolumn{4}{|l|}{ Kompetenz } \\
\hline Kompetenzen dokumentieren & 85 & Kompetenzmatrix & 87 \\
\hline Formelle Weiterbildung & 89 & Weiterbildungskurse & 91 \\
\hline Informelle Weiterbildung & 93 & Job Rotation & 95 \\
\hline Rekrutierung & 97 & Altersspezifische Rekrutierung & 99 \\
\hline Nachfolge gestalten/Wissenstransfer & 101 & Altersgemischte Tandems & 103 \\
\hline \multicolumn{4}{|l|}{ Werte, Einstellungen, Motivation } \\
\hline Anerkennungskultur & 109 & Feedback-Gespräche & 111 \\
\hline Schaffung gemeinsamer Werke & 113 & $\begin{array}{l}\text { Entwicklung einer Unternehmenskultur } \\
\text { („Corporate Culture“) }\end{array}$ & 115 \\
\hline Partizipation & 117 & Mitarbeiterbefragungen & 119 \\
\hline Motivation & 121 & Flexibilisierung von Arbeitszeit & 123 \\
\hline \multicolumn{4}{|l|}{ Arbeit, Arbeitsorganisation, Führung } \\
\hline $\begin{array}{l}\text { Anpassung der Arbeitsorganisation und } \\
\text {-inhalte }\end{array}$ & 131 & $\begin{array}{l}\text { Interner Stellenwechsel } \\
\text { Alter(n)sgerechte Stellenbeschreibun- } \\
\text { gen }\end{array}$ & $\begin{array}{l}133 \\
134\end{array}$ \\
\hline Alter(n)sgerechte Schichtmodelle & 135 & Vorwärtsrotation von Schichtplänen & 137 \\
\hline $\begin{array}{l}\text { Lebenslauforientierte Arbeitszeit- } \\
\text { modelle }\end{array}$ & 139 & (Lebens-)Arbeitszeitkonten & 141 \\
\hline Alter(n)sgerechte Führung & 143 & Sensibilisierung von Führungskräften & 145 \\
\hline
\end{tabular}

\section{Der Methodenkasten für die Demografiearbeit}

Während Großunternehmen schon länger ihre Maßnahmen zum demografischen Wandel durchführen oder zumindest in der Schublade haben, tun sich kleine und mittlere Unternehmen mit dem Thema noch schwer. Das Bundesministerium für Arbeit und Soziales hat deshalb einen Projektbericht veröffentlicht, in dem die Werkzeuge für die betriebliche Demografiearbeit systematisiert werden: Instrumentenkasten für eine altersgerechte Arbeitswelt in kleineren Organisationen und Unternehmen. Die 169-seitige Broschüre gibt einen fundierten Überblick über mögliche innerbetriebliche Maßnahmen zur Schaffung einer altersgerechten Arbeitswelt in Form eines Instrumentenkastens. Dieser ist nach zentralen Handlungsbereichen zur Sicherung der Arbeitsfähigkeit gegliedert: Gesundheit, Kompetenz, Motivation und Arbeitsorganisation. Die beispielhaft dargestellten Instrumente werden jeweils nach Zielen und Gelingensbedingungen, Nutzen und Kosten sowie nach Umsetzungsvarianten analysiert, ergänzt durch Praxisbeispiele. Der Forschungsbericht steht kostenlos im Internet zum Herunterladen zur Verfügung.

www.bmas.de (Stichwort Forschungsbericht 424)

\section{Betriebliches Gesundheitsmanagement}

Unter dem Dach der Diakonie Michaelshoven gab es in der Vergangenheit schon zahlreiche Aktivitäten, die jedoch in diesem Jahr durch die Einführung eines unternehmensweiten betrieblichen Gesundheitsmanagements (BGM) gebündelt und qualifiziert werden. Das Unternehmen verpflichtet sich damit selbst und den Mitarbeitenden gegenüber in folgender Weise:

Mit der Einführung eines Betrieblichen Gesundheitsmanagements will die Diakonie Michaelshoven Gesundheit und Leistungsfähigkeit der Mitarbeitenden stärken: durch die gesundheitsförderliche Gestaltung der Arbeit und ihrer Organisation. Davon wird kein Geschäftsfeld ausgenommen!

Das Betriebliche Gesundheitsmanagement wird dazu beitragen, die Motivation der Mitarbeitenden zu stärken, die Kommunikation zu verbessern und die Identifikation mit der Diakonie zu erhöhen. Gesundheitsprävention soll zum Bestandteil gelebter Unternehmenskultur werden.

Diese Ziele sollen durch die dauerhafte Integration des Gesundheitsmanagements in betriebliche Prozesse und Strukturen erreicht werden. Die Diakonie Michaelshoven wird die dafür notwendigen betrieblichen Rahmenbedingungen schaffen.

Die Arbeitsstruktur des Betrieblichen Gesundheitsmanagements, derer sich die Diakonie Michaelshoven bedient, erfolgt analog zu vielen geschäftsfeldübergreifenden Prozessen. Ein zentraler Arbeitskreis führt die Anliegen der dezentralen Gesundheitszirkel der Geschäftsfelder zusammen und pflegt eine enge Rückkopplung mit den Leitungskreis aus Vorstand und Geschäftsführungen.

Seinen Weg in die Breite soll das Betriebliche Gesundheitsmanagement durch eine die Aufmerksamkeit der Mitarbeitenden bindende Kampagne (prominente Schirmherrschaft, kleiner Wettbewerb für eine »Gut-zu-Fuß«-Kampagne mit Schrittzählern als Give-away, pfiffiges Info-Material) nehmen.

Nach der ersten Implementierungsphase wird auch die Mitgliedschaft und Nutzung von thematischen Netzwerken, beispielsweise Health Region Network/ INQA/Europäisches Unternehmensnetzwerk/BzgA geprüft und erfolgen. 


\section{Geschäftsfeldspezifische Aktivitäten}

Auf diese bereits existierenden Maßnahmen und Programme kann das Betriebliche Gesundheitsmanagement aufbauen; beispielhaft seien genannt:

- Gesundheitszirkel mit dem Schwerpunkt »Gesundheitsprävention « und dem besonderen Inhalt »Erhalt der Arbeitsfähigkeit der älteren Arbeitnehmer (im Schicht- und Gruppendienst) «

- Gesundheitstag 2011 - offener Tag mit kostenfreien Beratungs-, Wellness- und Schnupperangeboten für alle Mitarbeitenden

- Öffnung einiger Sportangebote und Sporträume des Berufsförderungswerkes für alle Mitarbeitenden

- Angebote zur kollegialen Beratung, Supervision und interne Beratungsund Unterstützungsmöglichkeiten durch eigene Fachdienste (Psychosozialer Dienst) im Bereich der Kinder-, Jugend- und Familienhilfen

\section{Eigenverantwortung}

Die Mitarbeitenden werden sensibilisiert für die Bedeutung der Eigenverantwortung für ihre Gesundheit. Auch dieser wichtige Baustein - wenn nicht sogar das Fundament auf dem Weg zu gesunden Mitarbeitenden - ist in den bisher bestehenden Angeboten bereits vorhan- den. So wurde zentral eine Vorteilsmitgliedschaft bei einem Fitness-Anbieter ausgehandelt, die vielen Mitarbeitenden an verschiedenen Standorten in Köln und Umgebung zugänglich ist. Gesunde Ernährung wird erleichtert durch die Vermittlung einer Gemüsekiste zu günstigen Konditionen (u. a. Lieferung an den Arbeitsplatz). Beide Angebote sind für den Arbeitgeber übrigens nicht mit Kosten verbunden. Und schließlich eine jährliche Aktion, die zugleich einen hohen Spaßfaktor aufweist und das WirGefühl stärkt: die zentrale Organisation und Teilnahme an Firmenläufen (Run of colors, Kirche läuft).

\section{Arbeitgeber-Beauftragten- Systeme}

Auch diese Systeme gehören zu den Instrumenten, die das gesundheitliche und seelische Wohl der Mitarbeitenden in den Mittelpunkt stellen und dabei auch präventive Ziele verfolgen. $\mathrm{Zu}$ nennen sind insbesondere die betriebliche Suchtkrankenhilfe sowie die Bereitstellung von Mobbing-Schlichtern, beides Angebote, die sukzessive geschäftsfeldübergreifend eingeführt werden sollen und betroffenen Mitarbeitenden fachgeschulte Kolleginnen und Kollegen mit besonderem Vertrauensstatus an die Seite stellen.

$\mathrm{Zu}$ diesem Beauftragten-System gehört auch das betriebliche Eingliederungsmanagement (BEM), welches alle
Maßnahmen umfasst, die dazu dienen, Mitarbeitende mit gesundheitlichen Problemen oder Behinderung dauerhaft an einem geeigneten Arbeitsplatz einzusetzen. Basierend auf dem $\mathbb{8} 84$ SGB IX, werden Mitarbeitende, die innerhalb eines Jahres länger als sechs Wochen (42

\section{Meilensteine für Vielfalt}

Auf dem Weg der Implementierung hat das Diversity-Management in der Diakonie Michaelshoven bereits einige Meilensteine hinter sich lassen können:

\section{8}

- Führungskräftetag

- Der Vorstand erläutert die Intention und gibt den Startschuss für den Prozess

- Beitritt der Diakonie Michaelshoven zur Charta der Vielfalt

\section{9}

- Festlegung der Struktur

- Benennung eines Diversitymanagers und Einrichtung einer Begleitgruppe

- Erste Maßnahmen

- Erste Bestandsaufnahme der drei für die Diakonie Michaelshoven priorisierten Diversity-Dimensionen

- Erarbeitung einer Konzeption

\section{0}

- Verabschiedung der ersten Konzeption und Namensgebung

- Umsetzung Patenschaftsmodell (ein Geschäftsfeld entwickelt modellhafte Konzepte und Maßnahmen; Piloten stehen vor unternehmensweiten Implementierungen) und Teilziele 2010

\section{Die Diakonie Michaelshoven e. V.}

ist der größte diakonische Träger im Kölner Raum. Rund 2.000 Mitarbeiter betreuen 3.500 Menschen in unterschiedlichen Einrichtungen der klassischen Arbeitsfelder der Diakonie. Die Diakonie Michaelshoven wurde 1950 gegründet, zunächst als Heim für Kriegswaisen. Heute ist sie unter der Leitung von Birgit Heide und Dr. Stefan Ziegler ein modernes, soziales Unternehmen mit entsprechenden Organisations- und Führungsstrukturen. Die Diakonie Michaelshoven umfasst die folgenden Geschäftsbereiche:

- Wohnen und Leben im Alter Michaelshoven gGmbH

- Kinder- und Familienhilfen Michaelshoven gGmbH

- Wohnen und Leben mit Behinderung Michaelshoven gGmbH

- Berufsförderungswerk Köln gGmbH (mit Berufskolleg)

- Die Sozialen Hilfen

- Auxilio Dienstleistungen Michaelshoven $\mathrm{GmbH}$

- Management Services Michaelshoven

- Stiftung einfach helfen

Die Zentrale, mit Vorstand, Verwaltung und vielen Einrichtungen, liegt in Michaelshoven in Köln-Rodenkirchen. Außenstellen gibt es über die Grenzen Kölns hinaus, von Mönchengladbach im Norden bis Bonn im Süden und dem Bergischen Land im Osten.

www.diakonie-michaelshoven.de
- Zukunftswerkstatt Migration - eine gemeinsame Veranstaltung für alle Geschäftsfelder und alle Personalebenen des Unternehmens

\section{$2011 / 2012$}

- Schwerpunktthema »Interkulturelle Öffnung «: Einrichtung eines Forums Perspektive Vielfalt im Intranet »dia. log«; Unternehmensweiter Workshop "Interkulturelle Öffnung und diakonisches Profil«; Verabschiedung der "Handlungsorientierung Interkulturelle Öffnung « - Ziele und Maßnahmenplan; Entwicklung eines Fortbildungspiloten für Führungskräfte

\section{$2013 / 2014$}

- Schwerpunkthema Demografische Entwicklung in enger Verbindung mit Betrieblichem Gesundheitsmanagement 
Tage) ununterbrochen oder wiederholt arbeitsunfähig gewesen sind, vom Arbeitgeber über die Möglichkeiten aufgeklärt:

- wie die Arbeitsunfähigkeit möglichst überwunden werden kann

- mit welchen Leistungen oder Hilfen erneuter Arbeitsunfähigkeit vorgebeugt und der Arbeitsplatz erhalten werden kann.

Die Teilnahme am betrieblichen Eingliederungsmanagement ist für Mitarbeitende freiwillig und sie können von folgenden Angeboten eines Integrationsteams Gebrauch machen:

- medizinische Rehabilitation

- behindertengerechte Arbeitsplatzgestaltung (Einsatz von Hilfsmitteln)

- Umsetzung auf einen anderen Arbeitsplatz

- Schulung und Qualifizierungsmaßnahmen

\section{Angebote im internen Fortbildungsprogramm}

Zuletzt sind noch einige Titel aus dem aktuellen Fortbildungsprogramm zu nennen, deren Teilnahme für die Mitarbeitenden in der Regel kostenfrei ist, wie zum Beispiel die Kurse: Kinästhetik, »Burnout und die Folgen: Wer ausbrennt muss einmal entflammt gewesen sein «, »Gesunder Rücken in Beruf und Alltag«, »Ich bin doch nicht im Stress! «, »Stressbewältigung und Entspannung «. Die Schulung in diesen Fortbildungen erfolgt überwiegend durch interne Fachkräfte, beispielsweise aus dem unternehmenseigenen Fachseminar für Altenpflege oder angestellten Psychologen.

\section{Ausblick}

Viele Aspekte, ein Ziel: gesunde Mitarbeitende und Erhalt ihrer Arbeitsfähigkeit. Um diesem Anspruch gerecht zu werden, bedarf es vielfältiger Aktivitäten auf allen Ebenen eines Unternehmens. Hilfreich ist immer, auf vorhandene Stärken, Ideen und bereits vorhandene Fachkräfte in den eigenen Reihen aufzubauen. Gleichzeitig bedarf es eines klaren Auftrags in Verbindung mit einer Zusammenführung und Koordination, damit die vielen Fäden eines so komplexen Feldes wie dem der Gesundheit von Mitarbeitenden zu einem starken Strang verwoben werden können und sich nicht verknoten. Diese sollten sich nicht verheddern, sondern verzahnen!

\section{Masterplan Gesundheitswesen}

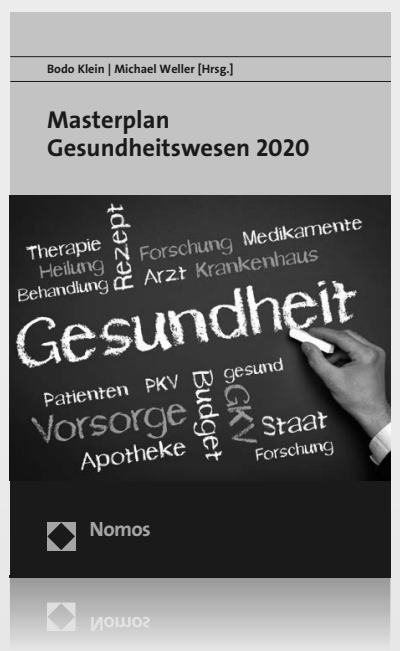

Was muss sich verändern, damit das deutsche Gesundheitswesen zukunftsfähig wird? Die Zeit ist reif für einen Masterplan Gesundheitswesen 2020. Das Buch analysiert in klaren Worten den Status quo und zeigt Wege zu einem zukunftsfesten Gesundheitswesen auf. Die Herausgeber fordern eine gesamtgesellschaftliche Kraftanstrengung nach den nächsten Bundestagswahlen.

Weitere Informationen: www.nomos-shop.de/13943

\section{Masterplan Gesundheitswesen 2020}

Herausgegeben von Dr. Bodo Klein und Michael Weller

2012, 225 S., brosch., 39,- -

ISBN 978-3-8329-6875-5

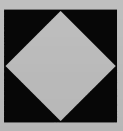

Nomos 